\title{
MODELING OF INTERSTELLAR DUST GRAINS
}

\author{
Nagalakshmi A. Rao*
}

\begin{abstract}
Dust has two major effects on light passing through the Interstellar Medium - Interstellar Extinction and Reddening. Interstellar dust grains are typically a fraction of a micron, approximately the wavelength of blue light and hence light passing through dust is depleted in blue wovelength, causing Interstellar Reddening. Dust Grain Models are mainly based on the analysis of intersfellar extinction and polarization curves. Discrefe Dipole Approximation (DDA) is the best studied model to compute scattering parameters of the grain.
\end{abstract}

Key words: Inferstellar medium (ISM), extinction, optical reddening, Discrefe dipole approximation (DDA)

\section{Introduction}

One of the most interesting facts about the evolution of the universe and the galaxy lies in the understanding of the Interstellar Medium - the vast volume between the stars. The interstellar medium is an active and dynamic zone and is a region of potential activity. The birth and death of stars takes place in this cool, low density region. Interestingly, the interstellar medium is not empty; it is enriched with gas \footnotetext{
Department of Physics, Government Science College, Bangalore 560 001, Karnataka, INDIA.
Email: drnarao@gmail.com
} 
(99\%) and sparsly dust (1\%). The exact nature and origin of interstellar dust grains is not known, however it is strongly believed that they are ejected from stars.

Dust has two major effects on light passing through the ISM. The starlight is dimmed by the dust, leading to Interstellar Extinction. Interstellar dust grains are typically a fraction of a micron, approximately the wavelength of blue light. Hence, light passing through dust is depleted in blue wavelength, causing Interstellar Reddening. Other important diagnostics for dust are emission from dust, scattering and polarization. Dust grain models are simulated which are mainly based on the analysis of interstellar extinction and polarization curves.

\section{Diagnostics of Interstellar Dust}

The striking evidence for the existence of interstellar dust comes from various lines [1 - 3]. When light passes through the interstellar medium, any one of the three effects occurs depending upon the grain size.

1) If dust is thick, light will be completely blocked, leading to dark areas called 'dark nebulae'.

2) The starlight is dimmed by dust, which is known as interstellar extinction.

3) Light that passes through dust is depleted in blue wavelength, resulting in interstellar reddening.

\section{Interstellar Extinction}

Whenever electromagnetic radiation propagates through a medium containing small particles like dust grains, dimming of starlight occurs. This phenomenon, known as Extinction, is the combined effect of absorption and scattering. Extinction is the best-studied property of diffuse dust and outer-cloud dust. This is because it can be determined accurately over a wide range of wavelength and for lines of sight sampling different physical conditions in the ISM.

The size of the dust particles in the ISM is typically a fraction of a micron, because of which scattering of blue light is favoured. Hence light which reaches us is more red in colour. This is known as Interstellar Reddening or Optical Reddening. Interstellar Extinction curve shows a plot of the variation of the extinction with the wavelength of the incident light. This phenomenon extends into the ultraviolet and attenuation as a function of the wavelength gives information about the target dust particle. The attenuation increases from red to blue in the visible band and reaches 
a maximum at $2175 \mathrm{~A}^{\circ}$ in the ultraviolet. The $2175 \mathrm{~A}^{\circ}$ bump is attributed to small $C$ and carbonaceous particles present in the dust.

\section{Interstellar Scattering}

Scattering provides a vital clue on the nature and composition of dust. Scattering can be observed in three general situations:

1. The Diffuse Galactic Light (DGL) - ie. Scattering by diffuse dust

2. Reflection Nebulae

3. Scattering of interstellar radiation field by dark cloud

Since the geometry of grains is uncertain, one attempts to determine only two quantities characterizing the scattering process, namely

1. Albedo - fraction of the extinction that is scattering

2. the mean value of the cosine of the angle of scattering $(g)$

For isotropic scattering, $\mathrm{g}=0$ and for completely forward throwing scattering $\mathrm{g}=1$.

\section{Interstellar Polarisation}

Interstellar polarization arises from the propagation of electromagnetic radiation through the ISM containing aligned elongated interstellar grains. The magnetic field in the ISM is responsible for aligning the grains. Polarisation arises from the differential extinction of starlight by aligned grains. The extent of polarization depends on

1. the degree of alignment of grains with the local magnetic field

2. angle between the local magnetic field and the line of sight

3. variation of magnetic field along the line of sight.

In the optical region, light reaching us from reddened stars is linearly polarized because the extinction depends on the polarization mode. Interestingly, the aligned dust grains that polarize starlight will also produce linearly polarized infrared emission. Thus polarization and the accompanying emission in infrared are important diagnostic tools for understanding the optical properties of grains. It has been recently reported in literature that models based on emission aspects require the inclusion of PAHs. 


\section{Numerical Recipes}

The scattering of light by interstellar dust, also known as Interstellar Extinction or Optical Reddening is a well-known phenomenon that extends into the ultraviolet $[4-5]$. The wavelength-dependent extinction is one of the characteristic features of dust grains. Spectral analysis infers that the attenuation increases from red to blue in the visible band and reaches a maximum at $2175 \mathrm{~A}^{\circ}$ in the ultraviolet. This salient feature of the interstellar extinction curves for several stars with a typical so-called bump at a constant wavelength of about $220 \mathrm{~nm}$ is a diagnostic tool for analyzing the host particle - namely the scatterer. The $2175 \mathrm{~A}^{\circ}$ indicates that the dust particles distributed in the ISM are quite small in comparison with the wavelength of visible radiation and primarily composed of small Carbon and carbonaceous particles.

Theoretical models to account for observed extinction require large number of extremely small dust grains. It is commonly accepted that the fraction of vacuum in the interstellar dust is large. These appear as void or inclusions in the dust model. Carbonaceous material is predominantly carbon by mass including diamond, graphite, amorphous or glossy carbon, aliphatic hydrocarbons and / or PAHs. Studies reveal PAHs produce negligible scattering, but experimental observations and spectral diagnosis infer that scattering is predominant in grains. Thus it is impossible to construct a unique model satisfying the various observed phenomena consistent with theoretical considerations.

\section{Model Formulation}

Physical grain models are important in understanding the nature and composition of dust in the ISM. Dust determines the shape of the galaxy and is significant in the process of star formation. Dust Grain Models are mainly based on the analysis of interstellar extinction and polarization curves. Theoretical models to account for the observed extinction require a large number of extremely small uniformly sized particles - preferably spherical in shape and having a uniform distribution. However, such an ideal situation with identical particles having homogeneous distribution is highly uncommon in the outer space.

Among the several numerical recipes for dust models, the three best tested recipes are
1) Mie Theory
2) Effective Medium Theory (EMT)
3) Transition Matrix ( $T$ - matrix) 
Each of the above theories has its own advantage and disadvantage in explaining the observed fits. Present models rely much on Discrete Dipole Approximation method for treating the observed interstellar extinction.

\section{Mie Theory}

Mie Theory treats dust grains as homogeneous spheres with sizes approximately of the order of $0.01 \mu \mathrm{m}$ to explain scattering and absorption of light. In Mie theory, the scattered electromagnetic field and the field inside the particle are expanded as infinite series of independent solutions of the wave equations. The series coefficients are determined from the boundary conditions of the particle surface. Dust grains are assumed to have a complex refractive index given by $m=n+i k$. Mie theory is best applicable for those values of $m$ satisfying the condition $|m| x<1000$. Carbon and silicates are the materials most often used in cosmic dust models. Conventional Mie theory would not be strictly applicable for particles like graphite having anisotropic optical properties and is not a very good recipe for dust models.

\section{Effective Medium Theory}

Interstellar dust grains are not solid homogeneous materials, but are a mixture of inhomogeneous ingredients so that the dielectric function within them varies from one place to another. Inhomogeneity can occur in several ways in the dusty material.

1) Coagulation: If homogeneous and chemically identical particles coagulate at some instance during their evolution, the result will be the formation of a bigger particle with voids inside. The new grain formed will be heterogeneous and will have a fluffy structure. An additional dielectric function is needed for the description of such grains. If chemically diverse particles stick together, again a heterogeneous mixture is obtained.

2) Contamination: During grain formation tiny solid particles like PAHs or metal atoms are formed which tend to contaminate the grains and thereby introduce inhomogeneity.

The optical behaviour of composite grains is estimated based on the average dielectric function of the mixture. Effective Medium Theory (EMT) is an approach to treat inhomogeneous scatterers as homogeneous particles having an average or effective refractive index. The general condition of EMT applicability is that the size of the inclusion is small in comparison with the wavelength of the incident radiation. 


\section{Transition Matrix Formulation}

The Transition matrix formulation was first introduced by Waterman in 1965 to study electromagnetic scattering by single homogeneous non-spherical particles. T matrix technique is based on expanding the incident vector spherical wave functions (VSWFs) regular at the origin and expanding the scattered field outside a circumscribing sphere of a scatterer in VSWFs regular at far field region. Effectively, T-matrix is based on the linear operator method and transforms the expansion coefficients of the incident field into those of the scattered field. In T-matrix formulation, transition matrix elements are related to the components of the scattering amplitude. The incident field is assumed to be a plane wave and the scattered field is a superposition of the fields scattered by individual spheres. T-matrix can compute any scattering characteristic of a non-spherical particle and is suited for analyzing dust grains. However, this approach cannot be used to study inhomogeneous i.e., porous, composite and fluffy grains.

\section{Discrete Dipole Approximation}

Discrete Dipole Approximation (DDA) method was first proposed by Purcell and Pennypacker in 1973. in the DDA method, a composite grain of arbitrany shape is represented by an array of dipole elements. Each dipole has an oscillating polarization in response to both the incident radiation and the electric fields of the other dipoles in the array. The superposition of the dipole polarization leads to extinction and scattering cross-sections. Examples of DDA calculations are described by Draine (1988), Bazell and Dwek (1990) and Fogel and Leung (1998).

\section{Applicability of DDA}

In the Discrete Dipole Approximation Method, the target is replaced by a 3D array of $\mathrm{N}$ oscillating dipoles $[6-8]$. Each dipole not only responds to the external electric field of the medium, but also to the electric field of the remaining $N-1$ dipoles that comprise the grain. The polarization at each dipole site is coupled to all other dipoles in the grain.

It is assumed that the dipole separation dis small compared to

1) any structural lengths in the target

2) wavelength of light $\lambda$.

Numerical siudies indicate that the second criteria is satisfied if $|\mathrm{m}| \mathrm{kd}<1$ 
where $m$ is the complex refractive index of the target material and $k=2 \pi / \lambda$ is the wave number.

If the target is represented by an array of $\mathrm{N}$ dipoles located in a cubic lattice within a lattice spacing $d$, then the target volume is given by $V=\mathrm{Nd}^{3}$.

The size of the target is characterized by an effective radius, $a_{\text {eff }}$ given by $a_{\text {eff }}=(3 V / 2 \pi)^{1 / 3}$

A typical scattering problem [9 - 11] is characterized by a dimensionless "size

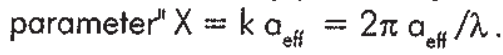

It is shown that

$$
A_{v}=R_{v} E_{B-v}
$$

where $E_{B . V}$ is the 'Colour Excess'.

$R_{v}$ is termed as the ratio of total to selective visual extinction. Theoretically, $R_{v}$ is expected to depend on the size and composition of interstellar dust grains. In low density $I S M, R_{v}$ is shown to be approximately a constant having a value $R_{v} \approx 3.05+0.15$.

The entire interstellar extinction curve is represented by the equation

$$
A_{\gamma} / A_{v}=a(x)+b(x) / R_{v}
$$

where $a(x)$ and $b(x)$ are the coefficients that have unique values at a given wave number, $x$. The $R_{v}$ dependent extinction is referred to as the CCM - extinction law.

Out of the 150 stars selected, we have computed $A_{\lambda} / A_{v}$ for various stars . Listed in Tablel are the values of $A_{\lambda} / A_{v}$ for few stars - HD190429 $\left(R_{v}=2: 4\right)$, HDI75514 $\left(R_{v}=2.8\right), H D 147707\left(R_{v}=4.0\right), H D 168021\left(R_{v}=5.0\right)$ and HD $38087\left(R_{v}=5.3\right) . R_{v}$, the ratio of total to selective extinction depends on the line of sight. Studies infer that in diffuse ISM, $R_{v} \approx 3.1$, but is anomalously large $R_{v} \approx 5.3$ in certain other regions of the interstellar medium. In the present study, we have selected stars with $R_{v}$ ranging from a value 2.4 for HD190429 to 5.3 for HD38087 thus spaning a large volume of the interstellar space. The large values of $R_{v}$ have been attributed to large grain size. In these regions of anomalous extinction, the dust grains are likely to be irregular in shape and fluffy in structure. The gradation in property from diffuse dust to inner cloud dust is a striking feature of the validity of the model. 
Furthermore, for the selected stars, it is observed from Table 1 that for wavelengths $\lambda>0.70 \mu \mathrm{m}$, the function $A_{\lambda} / A_{v}$ is approximately "universal", meaning that it is independent of $R_{v}$ This infers that for any $\lambda$ in the ultraviolet region, the interstellar extinction is independent of $R_{v}$.

DDSCAT code is highly flexible and can be applied to a wide variety of scattering situations by grains having complex refractive index, defined by $m=n+i k$. The refractive index is an indicator of the refractory elements in the constituent grains and ranges from very large values to small values. Standard values of $m$ are listed below

a. $m=$ infinity corresponds to an infinite dielectric constant

b. $m=1.33$ for 'ice' particles at visual wavelength

c. $m=1.33-0.09$ i for 'dirty ice' - i.e. ice with absorbing impurities

d. $m=1.27-1.37 \mathrm{i}$ corresponds to Fe.

The extinction efficiency factor, $Q_{\text {ext }}$, is defined as the ratio of extinction, crosssection to the geometric cross-section. Also $Q_{\text {ext }}$ is the sum of the corresponding efficiency factors for absorption and scattering and is given by

$$
Q_{e x t}=Q_{a b s}+Q_{s c a}
$$

These efficiencies are functions of two quantities, namely the dimensionless size parameter, $X=2 \pi \mathrm{a} / \lambda$ and the complex refractive index, $m$.

For pure dielectric materials, $k=0$. Ices and silicates are examples of astrophysically significant solids behaving approximately as dielectrics $(k<0.1)$ over much of the electromagnetic spectrum. On the other hand, for strongly absorbing materials such as metals, $k \approx n$.

Using the $D D A$ simulation, $Q_{s c a}, Q_{o b s}$ and $Q_{\text {ext }}$ can be computed for different values of the complex refractive index $m$. The main advantage of DDA is that it is one of the most general methods and does not require any symmetry aspects of the scatterer. However, it is seen that DDA is best suited for small values of $m$ and the Finite Difference Time Domain (FDTD) for large values of $m$. Basically DDA is best suited for scattering by dielectric targets whose sizes are comparable with the wavelength of light. 


\section{Conclusion}

A study of interstellar dust grains is essential in understanding the dynamical, thermal and chemical properties of the interstellar medium [12 - 15]. Satellites and probes are used to make observations of outer space and interstellar medium. Rapid progress is made in the study of the interstellar medium by successful performance of IUE (International UV Explorer), IRAS, COBE (Cosmic Background Explorer), HST (Hubble Space Telescope), ISO (Infrared Space Observatory) and various ground-based UV, optical and IR instruments. Cosmic dust grains are studied from Xrays to radio wavelengths. Dust has energy levels which are lowlying. Dust grains interact in many ways with the gas, the radiation and the magnetic field and hence can modify the structure of the stellar environment and influence its evolution. Such studies are theoretically challenging and potentially exciting and shed light on the evolution of the galaxy and universe.

\section{Acknowledgements}

The author is grateful to University Grants Commission for the financial support extended under the grant MRP(S) - 410/2006 (X Plan) / KABAOI6/UGC SWRO in the execution of this project.

Table 1. Interstellar Extinction for selected stars as a function of $\lambda$

\begin{tabular}{|c|c|c|c|c|c|c|c|c|}
\hline $\begin{array}{c}\lambda \\
(\mu \mathrm{m})\end{array}$ & $\begin{array}{c}\lambda^{-1} \\
\mu m^{-1}\end{array}$ & $a(x)$ & $b(x)$ & $\begin{array}{c}H D \\
190429 \\
R_{v}=2.4 \\
A_{2} / A_{v}\end{array}$ & $\begin{array}{c}H D \\
175514 \\
R_{v}=2.8 \\
A_{2} / A_{v}\end{array}$ & $\begin{array}{c}H D \\
147701 \\
R_{v}=4.0 \\
A_{\lambda} / A_{v}\end{array}$ & $\begin{array}{c}H D \\
168021 \\
R_{v}=5.0 \\
A_{2} / A_{v}\end{array}$ & $\begin{array}{c}H D \\
38087 \\
R_{v}=5.3 \\
A_{\lambda} / A_{v}\end{array}$ \\
\hline 0.125 & 8.00 & -1.0705 & 13.6700 & 4.6250 & 3.8116 & 2.3470 & 1.6635 & 1.5087 \\
\hline 0.150 & 6.67 & -0.4086 & 9.5342 & 3.5640 & 2.9965 & 1.9749 & 1.4982 & 1.3903 \\
\hline 0.180 & 5.56 & -0.0950 & 8.1091 & 3.2840 & 2.8011 & 1.9323 & 1.5268 & 1.4350 \\
\hline 0.2175 & 4.60 & 0.0003 & 9.8896 & 4.1210 & 3.5323 & 2.4727 & 1.9782 & 1.8662 \\
\hline 0.250 & 4.00 & 0.3581 & 6.0738 & 2.8889 & 2.5273 & 1.8765 & 1.5728 & 1.5041 \\
\hline 0.3125 & 3.20 & 0.7004 & 3.2792 & 2.0667 & 1.8715 & 1.5202 & 1.3562 & 1.3191 \\
\hline 0.360 & 2.78 & 0.9530 & 1.9090 & 1.7484 & 1.6348 & 1.4303 & 1.3348 & 1.1588 \\
\hline 0.440 & 2.27 & 0.9982 & 1.0495 & 1.4355 & 1.3730 & 1.2065 & 1.2081 & 1.1962 \\
\hline 0.550 & 1.82 & 1.0000 & 0.0000 & 1.0000 & 1.0000 & 1.0000 & 1.0000 & 1.0000 \\
\hline
\end{tabular}


Table 1 (Contd.)

\begin{tabular}{|c|c|c|c|c|c|c|c|c|}
\hline$\lambda$ & $\lambda^{-1}$ & $a(x)$ & $b(x)$ & $\begin{array}{c}H D \\
190429\end{array}$ & $\begin{array}{c}H D \\
175514\end{array}$ & $\begin{array}{c}H D \\
147701\end{array}$ & $\begin{array}{c}H D \\
168021\end{array}$ & $\begin{array}{c}H D \\
38087 \\
R_{v}=2.8 \\
R_{v}=4.0\end{array}$ \\
$A_{v}=5.0$ & $\begin{array}{c}A_{v} \\
R_{v}=5.3 \\
R_{v} / A_{v}\end{array}$ & $A_{\lambda} / A_{v}$ & $A_{\lambda} / A_{v}$ & $A_{\lambda} / A_{v}$ \\
\hline 0.700 & 1.43 & 0.8686 & -0.3660 & 0.7161 & 0.7379 & 0.7771 & 0.7954 & 0.7995 \\
\hline 0.900 & 1.11 & 0.6800 & -0.6239 & 0.4200 & 0.4571 & 0.5240 & 0.5552 & 0.5622 \\
\hline 1.250 & 0.80 & 0.4008 & -0.3679 & 0.2475 & 0.2694 & 0.3088 & 0.3272 & 0.3313 \\
\hline 1.600 & 0.63 & 0.2693 & -0.2473 & 0.1662 & 0.1810 & 0.2075 & 0.2198 & 0.2226 \\
\hline 2.200 & 0.46 & 0.1615 & -0.1483 & 0.0997 & 0.1085 & 0.1244 & 0.1318 & 0.1335 \\
\hline 3.400 & 0.29 & 0.0800 & -0.0734 & 0.0494 & 0.0538 & 0.0616 & 0.0653 & 0.0661 \\
\hline
\end{tabular}

\section{References}

1. D. C. B. Whittet, Dust in the galactic environment, $2^{\text {nd }}$ edn. IOP (2003).

2. Endrik Krugel, Physics of Interstellar Dust, loP (2003).

3. Dyson J. E. and Williams D. A, The Physics of the Interstellar Medium, 2nd edn. loP (1997).

4. Oort J. H and Van de Hulst H. C, Bull. Astro. Inst. Netherlands, 10, 187 (1946).

5. Mathis J. S, Rumpl W and Nordsieck K. H, ApJ, 217, 425 (1997).

6. Mathis J. S, ApJ , 497, 824 (1998).

7. Weingarner J. C and Draine B. T, ApJ, 548, 296 (2001).

8. Li A and Draine B. T, ApJ, 554, 778 (2001).

9. Vaidya D. B, Gupta R, Dobbie J. S and Chylek P, A \& A, 375, 584 (2001).

10. Clayton G. C. Wolff M. J, Sotia U. J, Gordon K. D and Misselt K. A, ApJ, 588, 871 (2003).

11. Michael I. Mishchenko, Travis L. D and Lacis A. A, Scattering, Absorption and Emission of light by small particles, Cambridge University Press (2002)

12. Modeling the stellar environment - How and Why? Proceedings of the fourth IAP Astrophysics Meeting, Editions Frontiers, 1988

13. James Lequeux, The Intersfellar Medium, Springer, New York (2005).

14. Millar T. J and Williams D A, Dust and Chemistry in Astronomy, loP (1993).

15. Jonathan Tennyson, Astronomical Spectroscopy - An introduction to the Atomic and Molecular Physies of Astronomical Spectra, Imperial College Press, (2005). 